\title{
Metamaterial Inspired Microstrip Antenna Investigations Using Metascreens
}

\author{
Muhammad Tauseef Asim ${ }^{1,2}$ and Mushtaq Ahmed ${ }^{1,2}$ \\ ${ }^{1}$ Pakistan Institute of Engineering and Applied Sciences (PIEAS), Islamabad 44000, Pakistan \\ ${ }^{2}$ National Institute of Lasers and Optronics (NILOP), Islamabad 44000, Pakistan \\ Correspondence should be addressed to Muhammad Tauseef Asim; tauseefasim@yahoo.com
}

Received 15 October 2014; Revised 10 February 2015; Accepted 11 February 2015

Academic Editor: Hui Feng Ma

Copyright (c) $2015 \mathrm{M}$. Tauseef Asim and M. Ahmed. This is an open access article distributed under the Creative Commons Attribution License, which permits unrestricted use, distribution, and reproduction in any medium, provided the original work is properly cited.

\begin{abstract}
A dual layer periodically patterned metamaterial inspired antenna on a low cost FR4 substrate is designed, simulated, fabricated, and tested. The eigenmode dispersion simulations are performed indicating the left handed metamaterial characteristics and are tunable with substrate permittivity. The same metamaterial unit cell structure is utilized to fabricate a metascreen. This metascreen is applied below the proposed metamaterial antenna and next used as superstrate above a simple patch to study the effects on impedance bandwidth, gain, and radiation patterns. The experimental results of these antennas are very good and closely match with the simulations. More importantly, the resonance for the proposed metamaterial antenna with metascreen occurs at the left handed (LH) eigenfrequency of the metamaterial unit cell structure. The measured $-10 \mathrm{~dB}$ bandwidths are $14.56 \%$ and $22.86 \%$ for the metamaterial antenna with single and double metascreens, respectively. The metascreens over the simple patch show adjacent dual band response. The first and second bands have measured $-10 \mathrm{~dB}$ bandwidths of $9.6 \%$ and $16.66 \%$. The simulated peak gain and radiation efficiency are $1.83 \mathrm{dBi}$ and $74 \%$, respectively. The radiation patterns are also very good and could be useful in the UWB wireless applications.
\end{abstract}

\section{Introduction}

Patch antennas have been the most demanded candidates for research activity due to their multiple advantages like low cost, low profile, being easy to fabricate, compact size, and conformability with the monolithic microwave integrated circuits (MMIC) [1,2]. It is the most widely used component in the wireless industry.

Metamaterials are artificially engineered homogeneous media with controllable electric and magnetic responses to give unusual and useful phenomena [3]. These materials are characterized by either dispersion relations or by constitutive electromagnetic parameters. The first formal theoretical studies on media with simultaneous double negative parameters were done by Veselago who predicted some novel phenomena inferred from these metamaterials in his famous paper [4]. After long time, Pendry el al. in 1990s demonstrated electric plasma (negative permittivity) by wire structures [5] and then magnetic plasma (negative permeability) by ring structures [6]. The first experimental demonstration for double negative structures was performed by Smith et al. [7]. Since the practical advent of metamaterials, these have been keenly studied as a potential artificial material for a number of applications in the microwave and optical region [8-11]. One such application is the substrate material for microstrip patch antennas. Researchers have investigated extensively to improve its features like return loss, VSWR, bandwidth, gain, and directivity [12-17].

The metamaterial substrates are realized based on 3D regular periodic arrangements of split ring resonator (SRR) and rod structures [18-22]. These types of substrates are bulky and very much lossy in the microwave region which hinders their use for the wireless applications [23]. Alternatively many researchers have studied planar transmission line metamaterials for antenna applications due to their ease of fabrication, compact size, and high efficiency [24-29]. 


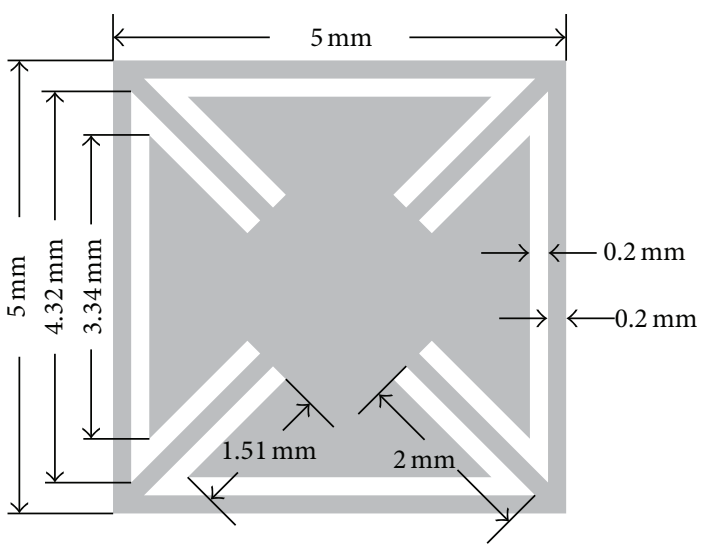

(a)

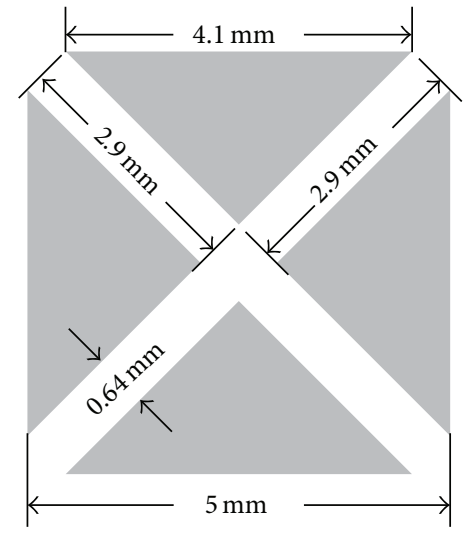

(b)

FIgURE 1: Unit cell: (a) top and (b) bottom dimensions.

There are many types of planar metamaterial based antennas [30], which are inspired from the metamaterial unit cell designs to enhance their performance. These planar metamaterial antennas can be broadly categorized as (i) dispersion engineered antennas [31-34], (ii) metamaterial unit cell loading antennas [35, 36], (iii) meta-resonator antennas [37, 38], and (iv) meta-surface loaded antennas [3942].

Previously Li et al. [31] demonstrated a patch antenna based on the modified form of the planar metamaterial structure proposed by Matsunaga et al. [43]. These structures were proposed to avoid the use of via hole in the unit cell for the ease of fabrication purposes. These structures were designed using left handed (LH) dispersion engineering approach. Recently Xiong et al. [44] also demonstrated a patch antenna based on similar structure with modified ground pattern for enhanced performance.

The aim of our work is to design, simulate, fabricate, and investigate a novel metamaterial antenna realized by the metamaterial unit cell structure proposed by Matsunaga et al. [43]. The same metamaterial structure is further utilized to fabricate a metascreen. The size of the metascreen is kept equal to the size of antenna to miniaturize the overall design. The proposed metascreen is used in the near field of the proposed metamaterial antenna and also over a simple patch antenna to investigate its effect on impedance bandwidth, gain, and radiation patterns.

\section{Geometrical Model}

The metamaterial unit cell is a dual layer planar copper structure on FR4 substrate for which the dimensions for the top and bottom layers are shown in Figure 1. The unit cell top layer consists of a small square copper patch of $(5 \times 5) \mathrm{mm}$ with disconnected triangular shaped slit gaps on the edges. The bottom layer consists of similar copper patch with cross slits, having gap width of $0.64 \mathrm{~mm}$. This unit cell is repeated twice in the $x y$ plane to generate the pattern of the patch antenna. The dimensions of the bottom and top antenna layers are shown in Figures 2(a) and 2(b), respectively. The substrate thickness is $1.51 \mathrm{~mm}$.

The proposed metamaterial (MTM) antenna is parametrically analyzed in commercial FEM based simulation software HFSS to get the optimum values for different antenna dimensions. The design gives FeedLoc $=0.5 \mathrm{~mm}$, Feed $L=4.2 \mathrm{~mm}$, Feed $W=3 \mathrm{~mm}, L=5 \mathrm{~mm}, W=10 \mathrm{~mm}$, $\operatorname{Sub} L=17.2 \mathrm{~mm}$, Sub $W=26 \mathrm{~mm}$, Sub $V=8 \mathrm{~mm}$, and GndGap $=0.5 \mathrm{~mm}$. Further we designed, simulated, fabricated, and investigated the MTM antenna and the simple patch antenna with metascreens made of the same metamaterial unit cell structure. Figure 3 shows the perspective views for the MTM antenna, MTM antenna with single and double metascreens. The gap between the antenna and the metascreen is also parametrically varied to investigate the effect of SubGap.

\section{Results and Discussions}

3.1. Dispersion Plots. Different eigenmode dispersion plots for the same structure on different substrate materials are shown in Figure 4. The left handed material (LHM) characteristic is indicated by the negative slope of these curves. The permittivity values of the substrates Roggers, FR4, and Duroid are $10.2,4.4$, and 2.2 , respectively, giving eigenfrequencies approximately around $6 \mathrm{GHz}, 9.1 \mathrm{GHz}$, and $11.5 \mathrm{GHz}$, respectively. The curves illustrate that the eigenmode response shifts towards the higher frequency side with the decrease of substrate permittivity. So permittivity variation keeps left handed characteristics of the curve intact but only scales the frequency response to the desired frequency band giving an option for substrate based tunability. All the curves give LH characteristics between points $X$ and $M$. The steepest descent makes a valley close to point $M$. These curve variations suggest the nonhomogeneous and anisotropic nature of the unit cell structure. The LH eigenfrequency band is very narrow confirming the band limited and dispersive nature of the metamaterial structures. The effect of substrate thickness is studied and it does not change the left handed characteristics of the curve. Interchanging the top and bottom structure in 


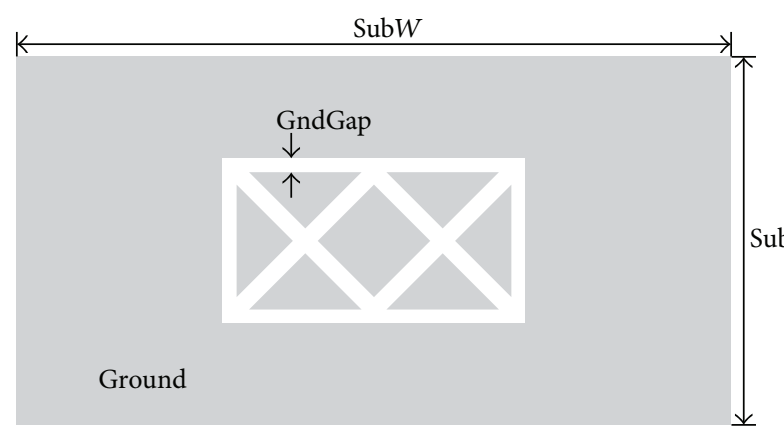

(a)

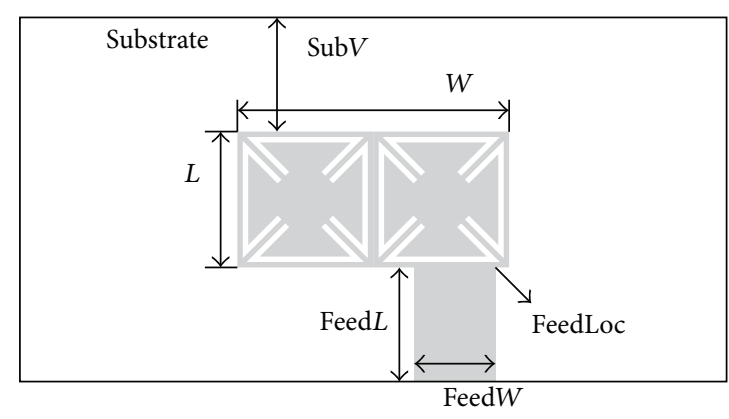

(b)

FIGURE 2: Patch antenna dimensions: (a) bottom view and (b) top view.

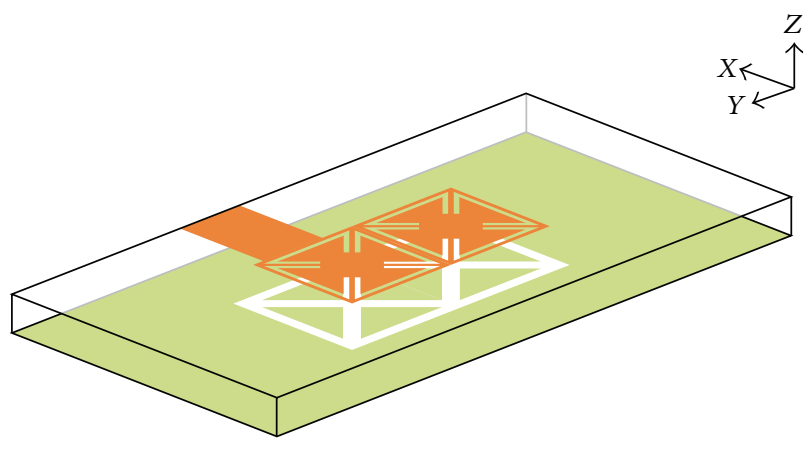

(a)

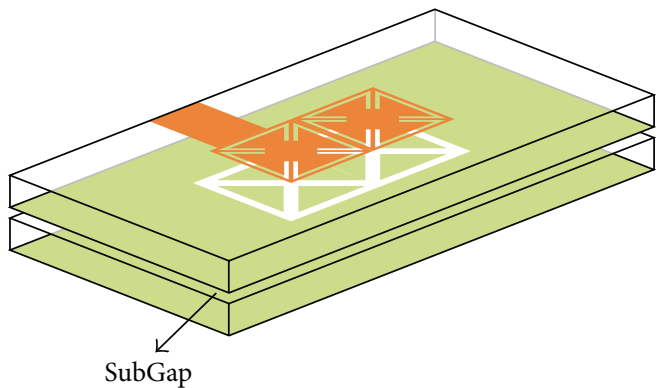

Gap between MTM antenna and metascreen

(b)

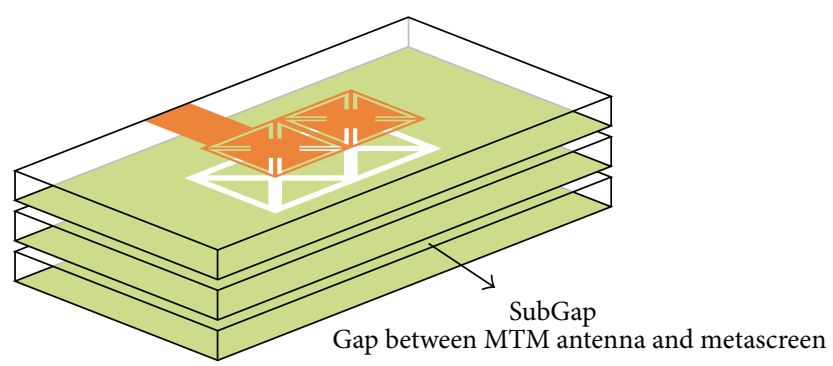

(c)

FIGURE 3: Perspective views of antennas with metascreens: (a) metamaterial (MTM) antenna, (b) MTM antenna with single metascreen, and (c) MTM antenna with double metascreen.

the unit cell also does not change the characteristics of the eigenresponse.

3.2. Simple Patch Antenna. A simple patch antenna with dimensions for length $L=5 \mathrm{~mm}$ and width $W=10 \mathrm{~mm}$ is designed, simulated, fabricated, and tested. The purpose is to compare the performance of simple patch antenna with MTM antenna and MTM antenna with metascreens, all having the same dimensions. This antenna gives resonance at $12.5 \mathrm{GHz}$ with off-centered feed location at FeedLoc $=$ $0.5 \mathrm{~mm}$, as shown in Figure 5(a). This is about $3 \mathrm{GHz}$ away from the eigenresponse. Plots for various FeedLoc are shown in Figure 5(a). The measured $-10 \mathrm{~dB}$ bandwidth is $8.4 \%$. The simulated peak gain and radiation efficiency are $3.9 \mathrm{dBi}$ and
$69 \%$, respectively. The fabricated antenna at $13 \mathrm{GHz}$ has a total size of $\left(0.216 \lambda_{0} \times 0.433 \lambda_{0} \times 0.065 \lambda_{0}\right)$.

3.3. MTM Antenna with Single and Double Metascreens. When we simulated the proposed MTM antenna having the dimensions $(5 \mathrm{~mm} \times 10 \mathrm{~mm})$ with FeedLoc at $0.5 \mathrm{~mm}$, it gave a resonance at $8.85 \mathrm{GHz}$, as shown in Figure $6(\mathrm{a})$ (solid line). The response is very weak but very close to the eigenresponse at $9.1 \mathrm{GHz}$. When we made simulation with a metascreen made of the same structure and size below this metamaterial antenna, it also gave resonance very close to the eigenfrequency but stronger than the simple MTM antenna, as shown in Figure 6(a) (dash line). The weak response is due to the lossy nature of these metamaterials and this fact 


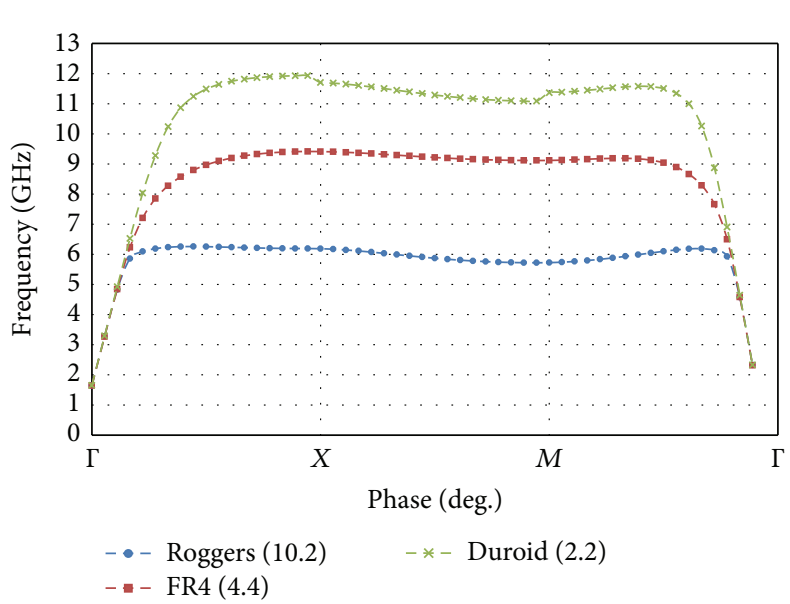

(a)

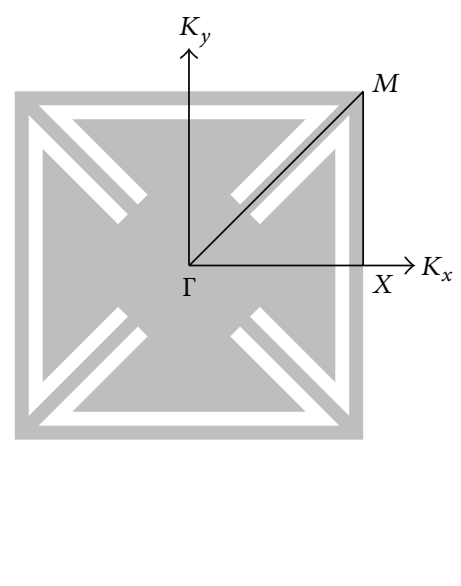

(b)

FIGURE 4: (a) Eigenfrequency dispersion plots for the metamaterial unit cell and (b) Brillouin zone showing the phase traversal path for dispersion plots in the $x y$-plane.

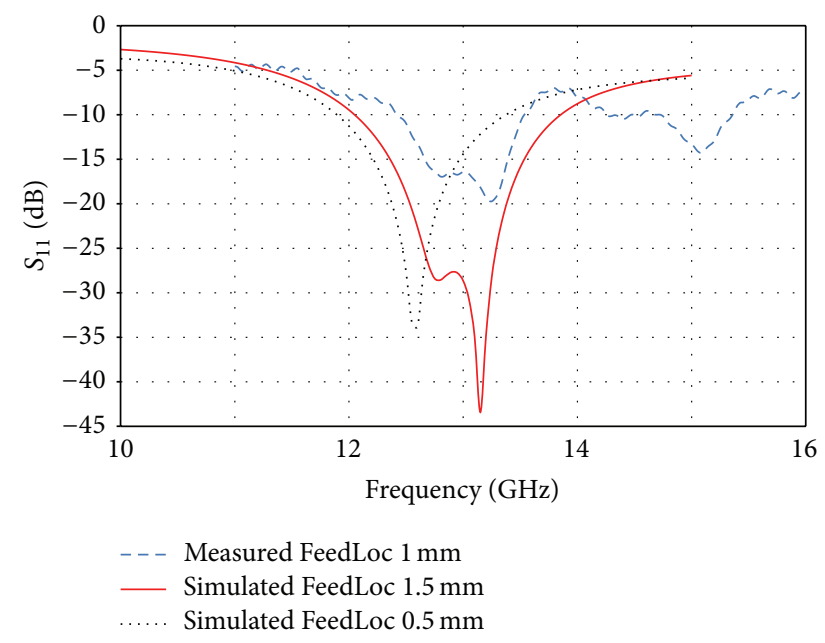

(a)

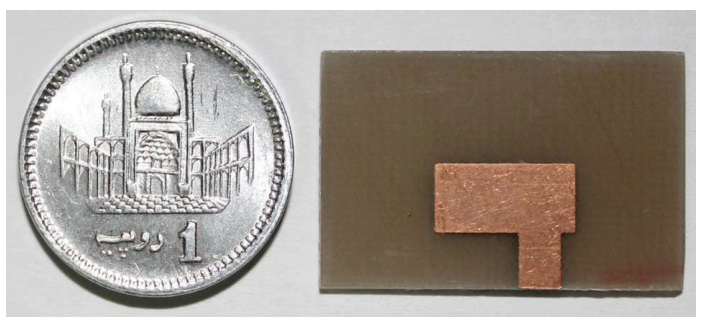

(b)

Figure 5: (a) Measured and simulated return loss for simple patch and (b) photograph for the fabricated simple patch $(5 \mathrm{~mm} \times 10 \mathrm{~mm})$; FeedLoc $=1 \mathrm{~mm}$.

is already reported in literature [30]. Another factor may be the loss tangent of FR4 material which is high at higher frequencies.

We fabricated the MTM antenna and metascreens. Experiments were performed by using single and then double metascreens below the metamaterial antenna. We got the measured resonance at the LH Eigenfrequency of $9.1 \mathrm{GHz}$ as shown and compared in Figure 7 with simulation results. The overall curve shapes are similar with some shift seen in the resonant frequencies. The reason for this frequency shift is due to the difference in boundary conditions [30]. The dispersion plots are obtained by using periodic boundary conditions while antennas use limited unit cells with radiation boundary conditions. Another factor is the permittivity variation of commercial FR4 substrates. It decreases with frequency and this causes the resonance to move towards higher side as depicted in Figure 7 (blue cross line). The comparison shows that the measured results for the single (solid line) and double metascreens (solid square line) under the MTM antenna closely match with each other in the frequency range from $8 \mathrm{GHz}$ to $9.5 \mathrm{GHz}$. However there is another resonance that appeared near $7.6 \mathrm{GHz}$ in the measured results for double metascreen which does not appear in the measured results for the single metascreen. The simulated results for the double metascreen are not shown since they follow the results for single metascreen case. The measured $-10 \mathrm{~dB}$ bandwidths at $9.1 \mathrm{GHz}$ are $14.56 \%$ (simulated $22 \%$ ) and $22.86 \%$ for the MTM antenna with single and double metascreens, respectively. The simulated peak gain and radiation efficiency are $0.7 \mathrm{dBi}$ and $90 \%$ for single metascreen case. The fabricated MTM antenna with single metascreen at $9.1 \mathrm{GHz}$ has a total size of $\left(0.152 \lambda_{0} \times 0.303 \lambda_{0} \times 0.106 \lambda_{0}\right)$. 


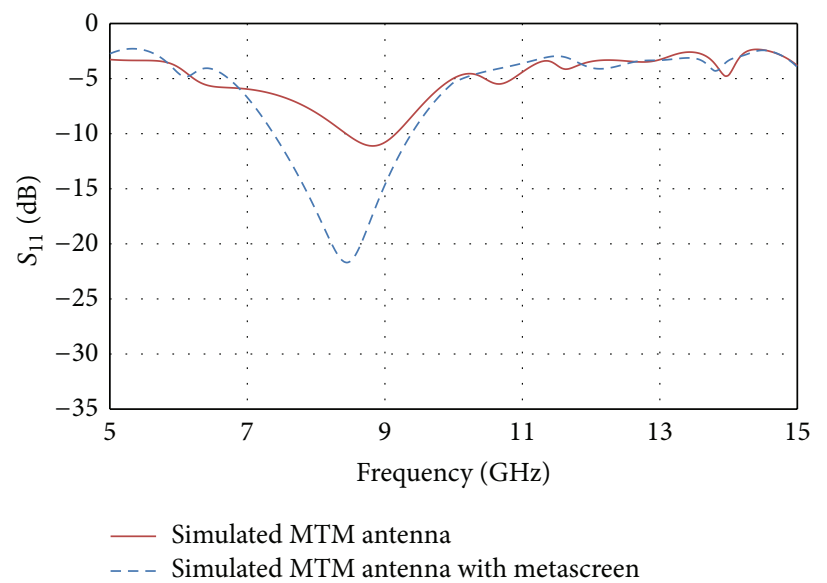

(a)

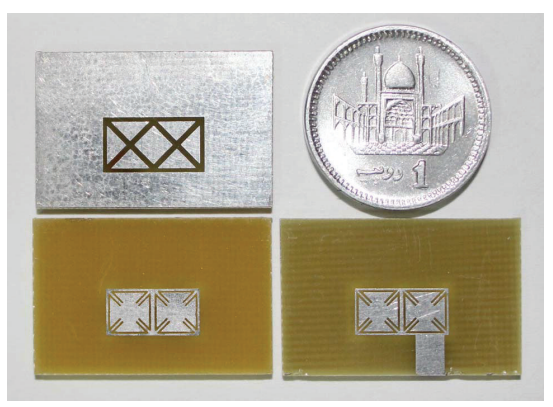

(b)

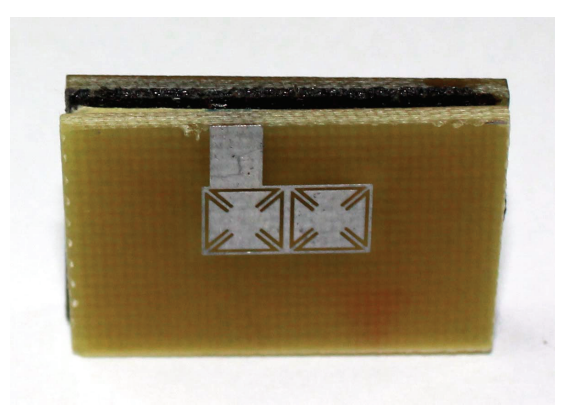

(c)

Figure 6: (a) Response for simulated MTM antenna and MTM antenna with single metascreen SubGap = $1.5 \mathrm{~mm}$. (b) Fabricated MTM antenna, metascreen top and bottom layers. (c) Side view showing the adhesive foam tape of $1.5 \mathrm{~mm}$ between MTM antenna and metascreen.

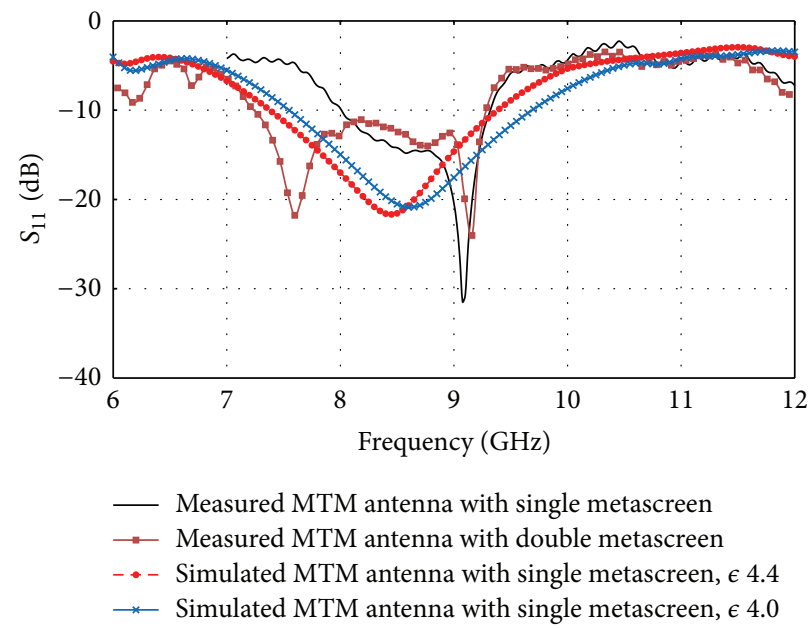

FIGURE 7: Return loss for metamaterial antenna with single and double metascreens.

3.4. Inverted Metascreen over Simple Patch Antenna. We performed more experiments by putting the metascreen slab inverted upside down over the simple patch antenna of the same size ( $5 \mathrm{~mm}$ by $10 \mathrm{~mm}$ ) as shown in the perspective view of Figure 8(b). As can be seen the measured resonances shown in Figure 5(a) of the simple patch at $13 \mathrm{GHz}$ and $15 \mathrm{GHz}$ have been changed as shown in Figure 9 (solid line) by using an inverted metascreen. The measured result is compared with the simulation results by varying the SubGap, that is, the gap between a simple patch and the metascreen. There are two adjacent resonances in the measured result with a small stopband of about $250 \mathrm{MHz}$. The first and second bands have measured $-10 \mathrm{~dB}$ bandwidths of $9.6 \%$ and $16.66 \%$ (21\% simulations). But these resonances are far away from 


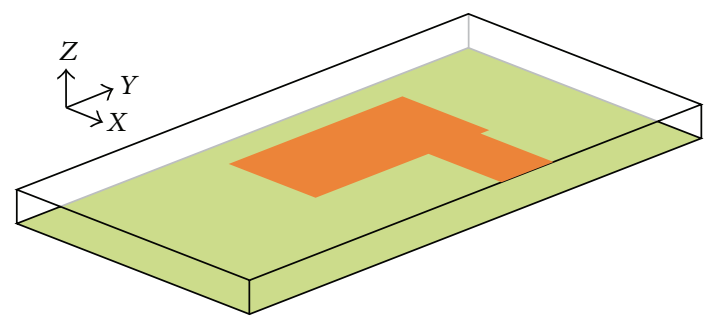

(a)

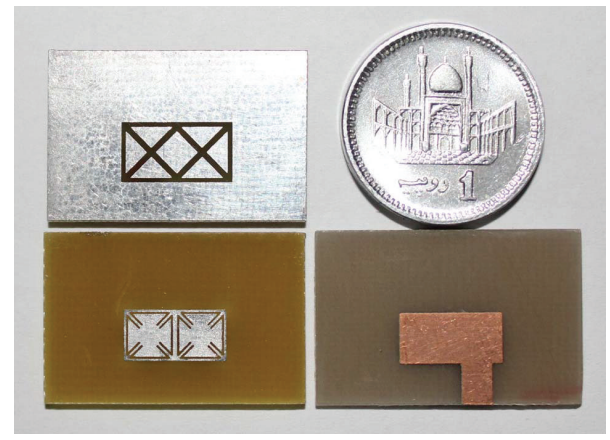

(c)

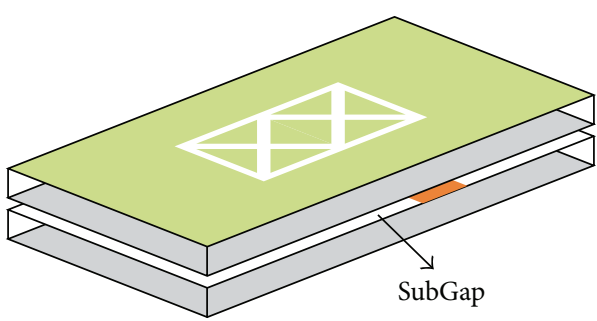

(b)

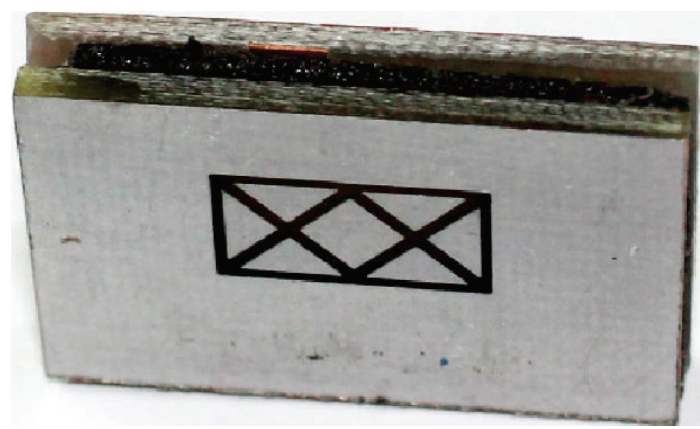

(d)

Figure 8: Perspective views for (a) simple patch antenna, (b) metascreen over simple patch antenna, (c) photograph for the fabricated simple patch antenna with metascreen bottom and top layers, and (d) side view.

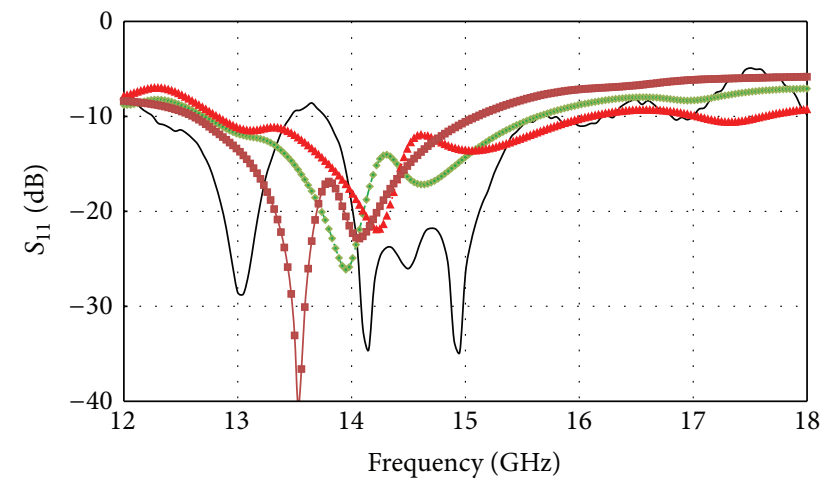

— Measured FeedLoc $1 \mathrm{~mm}$, SubGap $1.5 \mathrm{~mm}$

... Simulated FeedLoc $1 \mathrm{~mm}$, SubGap $1 \mathrm{~mm}$

- - Simulated FeedLoc 1mm, SubGap $1.5 \mathrm{~mm}$

$\rightarrow$ Simulated FeedLoc $1 \mathrm{~mm}$, SubGap $2 \mathrm{~mm}$

Figure 9: Measured and simulated return loss for inverted metascreen over simple patch antenna.

the eigenfrequency of the MTM unit cell, that is, $9.1 \mathrm{GHz}$. Nevertheless, these metascreens can be used to broaden the impedance bandwidths around the normal resonances of the simple patch antennas. The simulated peak gain and radiation efficiency are $1.83 \mathrm{dBi}$ and $74 \%$, respectively, at $13.95 \mathrm{GHz}$. The fabricated antenna with single metascreen at $13 \mathrm{GHz}$ has a total size of $\left(0.216 \lambda_{0} \times 0.433 \lambda_{0} \times 0.13 \lambda_{0}\right)$.

3.5. Radiation Patterns. The two-dimensional radiation patterns for the MTM antenna, MTM antenna with single metascreen, and simple patch with inverted metascreen are plotted in Figures 10, 11, and 12, respectively. The simple MTM antenna radiates at $8.85 \mathrm{GHz}$ which is mostly along the bore side and back side in the $x z$ plane while it is from 0 to -180 degrees in the $y z$ plane. The single metascreen under the MTM antenna also radiates along the bore side and back side in the $x z$ and $y z$ planes. The radiation pattern is also broadband as can be seen from response of two frequencies within the band. The radiation pattern for the metascreen over the simple patch is directional with maximum from -30 to -120 degrees in the $x z$ plane and 0 to -60 degrees in the $y z$ plane.

\section{Conclusion}

In conclusion a dual layer metamaterial inspired microstrip antenna on a low cost FR4 substrate is designed, simulated, fabricated, and tested. The metamaterial structure is dispersion engineered and its eigenfrequency is around $9.1 \mathrm{GHz}$ for the FR4 material. This eigenfrequency can be tuned to higher frequencies by using other substrate materials with lower permittivity values. This structure is utilized to fabricate a metascreen. This metascreen is applied under the proposed MTM antenna and also over a simple patch antenna to study their performance parameters such as impedance bandwidths, gains, and radiation patterns. The measured $-10 \mathrm{~dB}$ bandwidths at $9.1 \mathrm{GHz}$ are $14.56 \%$ (simulated $22 \%$ ) and $22.86 \%$ for the MTM antenna with single and double metascreens, respectively. The simulated peak gain and radiation efficiency are $0.7 \mathrm{dBi}$ and $90 \%$ for single metascreen case. The $-10 \mathrm{~dB}$ bandwidth for the simple patch with inverted metascreen is measured as $9.6 \%$ and $16.66 \%$ (21\% simulations) for dual band operation. The simulated 


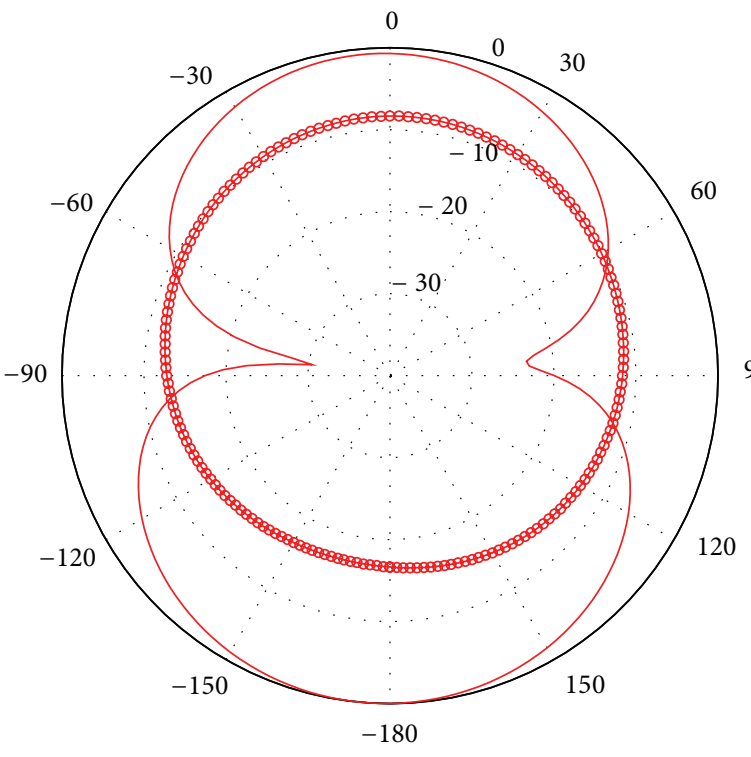

(a)

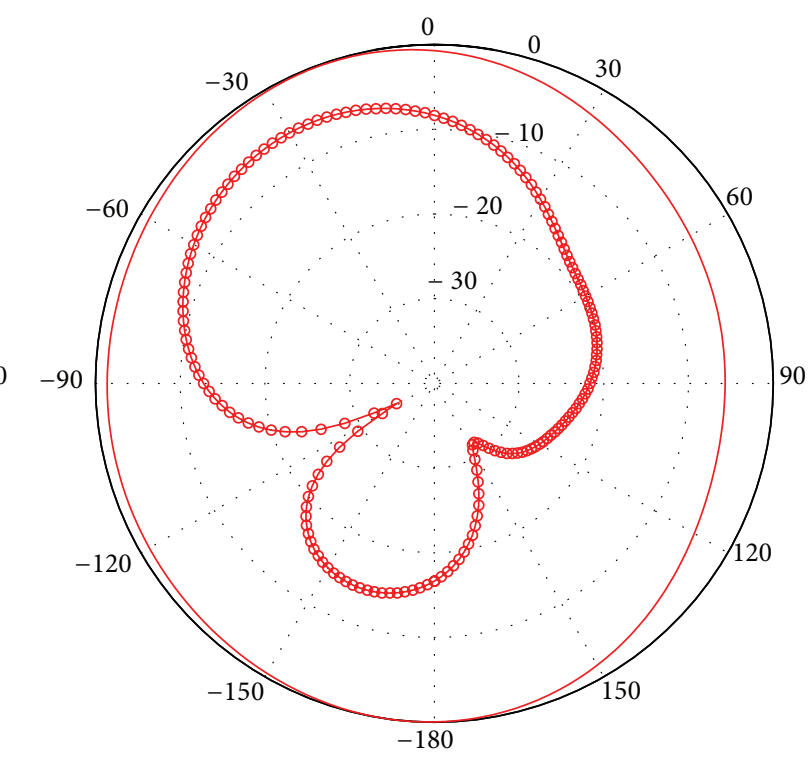

(b)

FIGURE 10: Simulated radiation patterns for MTM antenna, copolarization (solid line) and cross-polarization (solid circle line) at 8.85 GHz: (a) $x z$ plane and (b) $y z$ plane.

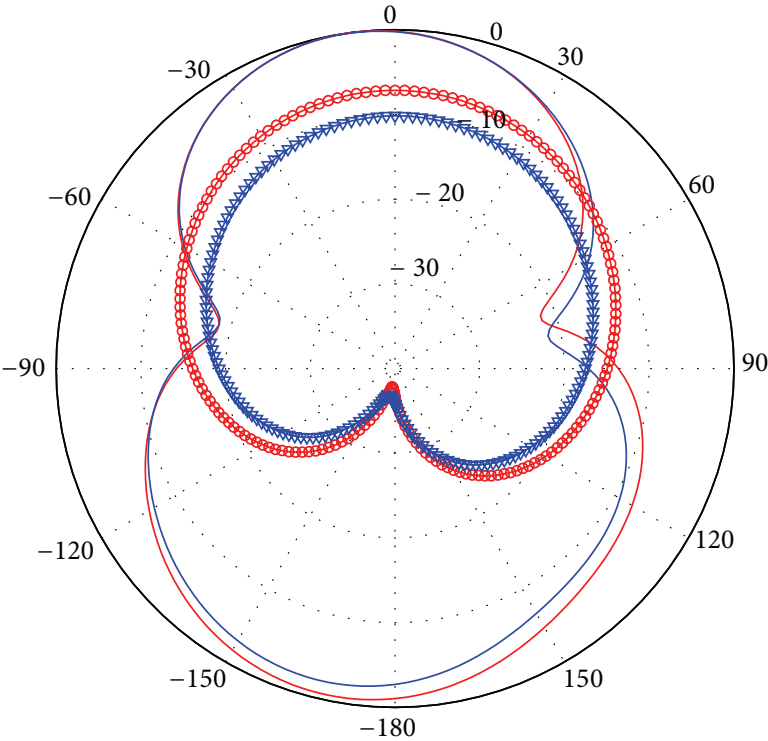

(a)

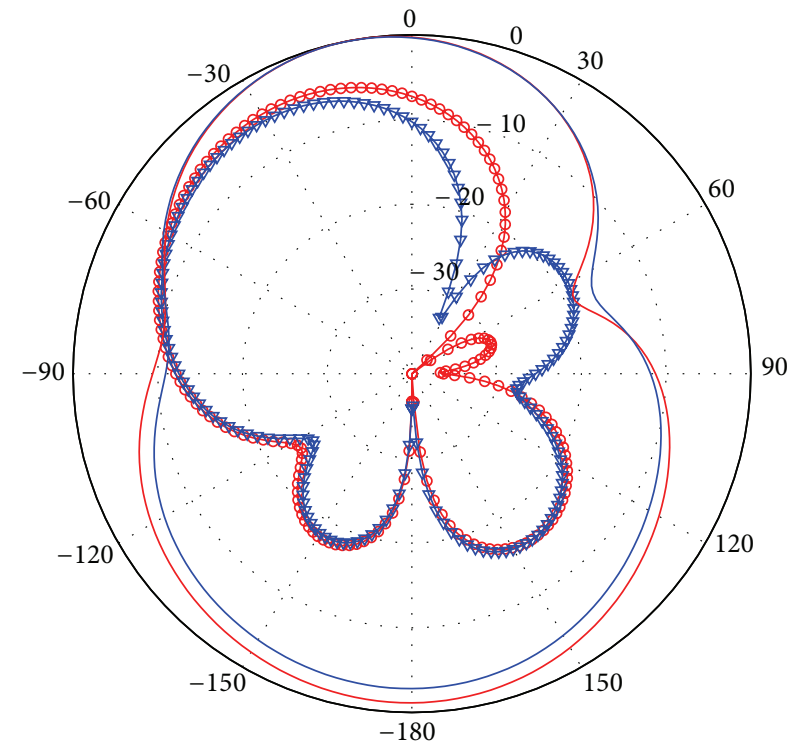

(b)

FIGURE 11: Simulated radiation patterns for MTM antenna with single metascreen, copolarization (solid red line) and cross-polarization (solid red circle line) at $8.45 \mathrm{GHz}$, copolarization (solid blue line) and cross-polarization (solid blue triangle line) at $9 \mathrm{GHz}$ : (a) $x z$ plane and (b) $y z$ plane. 


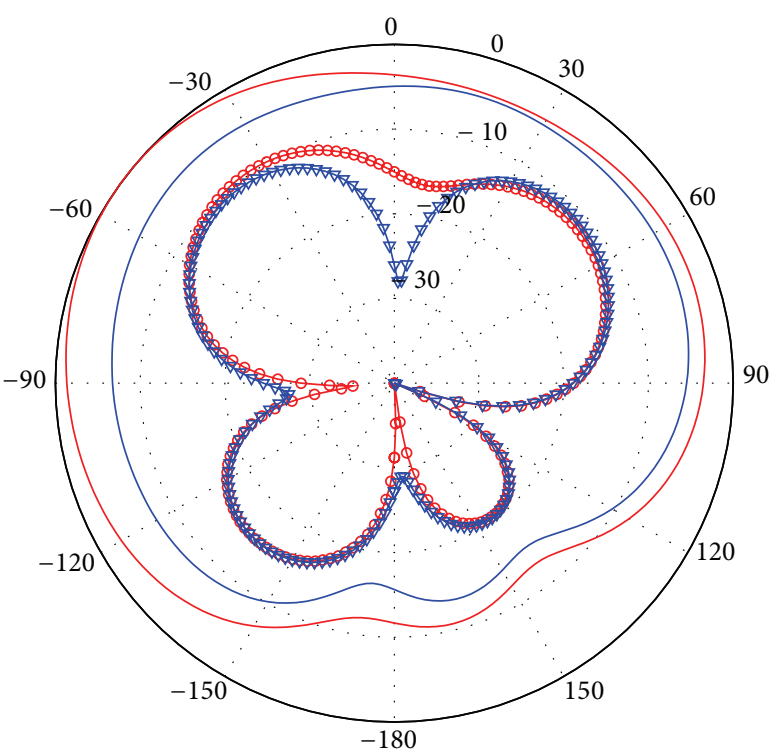

(a)

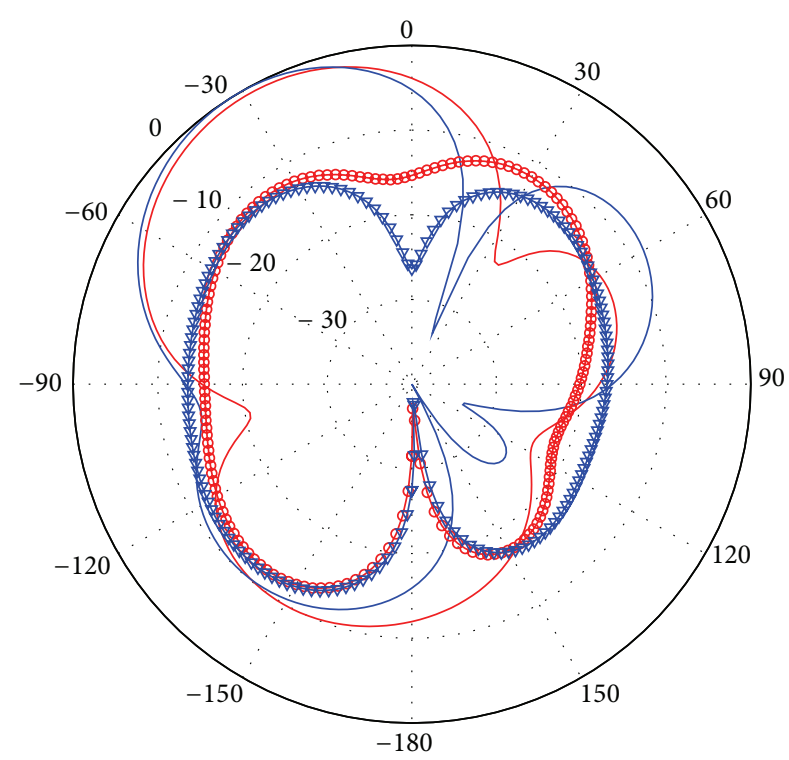

(b)

FIGURE 12: Simulated radiation patterns for simple patch with inverted metascreen, copolarization (solid red line) and cross-polarization (solid red circle line) at $13.95 \mathrm{GHz}$, copolarization (solid blue line) and cross-polarization (solid blue triangle line) at $14.64 \mathrm{GHz}$ : (a) $x z$ plane and (b) $y z$ plane.

peak gain and radiation efficiency are $1.83 \mathrm{dBi}$ and $74 \%$, respectively. The radiation patterns are also very good and could be useful for the UWB wireless applications.

\section{Conflict of Interests}

The authors declare that there is no conflict of interests regarding the publication of this paper.

\section{Acknowledgments}

This work is supported by the Higher Education Commission, Pakistan, through financial Grant no. 17-5-4(Eg4-032) $\mathrm{HEC} / \mathrm{Sch} / 2007$. The authors are indebted for providing the fabrication facility by the National Institute of Lasers and Optronics, Islamabad, Pakistan.

\section{References}

[1] C. A. Balanis, Antenna Theory: Analysis and Design, John Wiley \& Sons, Hoboken, NJ, USA, 2005.

[2] K.-L. Wong, Compact and Broadband Microstrip Antennas, Wiley, New York, NY, USA, 2002.

[3] M. Lapine and S. Tretyakov, "Contemporary notes on metamaterials," IET Microwaves, Antennas and Propagation, vol. 1, no. 1, pp. 3-11, 2007.

[4] V. G. Veselago, "The electrodynamics of substances with simultaneous negative values of $\varepsilon$ and $\mu$," Soviet Physics Uspekhi, vol. 10, no. 4, pp. 509-514, 1968.

[5] J. B. Pendry, A. J. Holden, W. J. Stewart, and I. Youngs, "Extremely low frequency plasmons in metallic mesostructures," Physical Review Letters, vol. 76, no. 25, pp. 4773-4776, 1996.
[6] J. B. Pendry, A. J. Holden, D. J. Robbins, and W. J. Stewart, "Magnetism from conductors and enhanced nonlinear phenomena," IEEE Transactions on Microwave Theory and Techniques, vol. 47, no. 11, pp. 2075-2084, 1999.

[7] D. R. Smith, W. J. Padilla, D. C. Vier, S. C. Nemat-Nasser, and S. Schultz, "Composite medium with simultaneous negative permeability and permittivity," Physical Review Letters, vol. 84, no. 18, pp. 4184-4187, 2000.

[8] N. Engheta and R. W. Ziolkowski, "A positive future for doublenegative metamaterials," IEEE Transactions on Microwave Theory and Techniques, vol. 53, no. 4, pp. 1535-1556, 2005.

[9] A. Alù, N. Engheta, A. Erentok, and R. W. Ziolkowski, "Singlenegative, double-negative, and low-index metamaterials and their electromagnetic applications," IEEE Antennas and Propagation Magazine, vol. 49, no. 1, pp. 23-36, 2007.

[10] N. Fang, H. Lee, C. Sun, and X. Zhang, "Sub-diffraction-limited optical imaging with a silver superlens," Science, vol. 308, no. 5721, pp. 534-537, 2005.

[11] J. B. Pendry, D. Schurig, and D. R. Smith, "Controlling electromagnetic fields," Science, vol. 312, no. 5781, pp. 1780-1782, 2006.

[12] C.-C. Chen and J. L. Volakis, "Bandwidth broadening of patch antennas using nonuniform substrates," Microwave and Optical Technology Letters, vol. 47, no. 5, pp. 421-423, 2005.

[13] J. Zhu and G. V. Eleftheriades, "A compact transmissionline metamaterial antenna with extended bandwidth," IEEE Antennas and Wireless Propagation Letters, vol. 8, pp. 295-298, 2009.

[14] B.-I. Wu, W. Wang, J. Pacheco Jr., X. Chen, T. M. Grzegorczyk, and J. A. Kong, "A study of using metamaterials as antenna substrate to enhance gain," Progress in Electromagnetics Research, vol. 51, pp. 295-328, 2005.

[15] B.-I. Wu, W. Wang, J. Pacheco et al., "Anisotropic metamaterials as antenna substrate to enhance directivity," Microwave and Optical Technology Letters, vol. 48, no. 4, pp. 680-683, 2006. 
[16] Z.-B. Weng, Y.-C. Jiao, G. Zhao, and F.-S. Zhang, "Design and experiment of one dimension and two dimension metamaterial structures for directive emission," Progress in Electromagnetics Research, vol. 70, pp. 199-209, 2007.

[17] H. Xu, Z. Zhao, Y. Lv, C. Du, and X. Luo, "Metamaterial superstrate and electromagnetic band-gap substrate for high directive antenna," International Journal of Infrared and Millimeter Waves, vol. 29, no. 5, pp. 493-498, 2008.

[18] N. Engheta and R. W. Ziolkowski, Metamaterials: Physics and Engineering Explorations, John Wiley \& Sons, Hoboken, NJ, USA, 2006.

[19] A. Erentok, P. L. Luljak, and R. W. Ziolkowski, "Characterization of a volumetric metamaterial realization of an artificial magnetic conductor for antenna applications," IEEE Transactions on Antennas and Propagation, vol. 53, no. 1, pp. 160-172, 2005.

[20] J. F. Woodley, M. S. Wheeler, and M. Mojahedi, "Left-handed and right-handed metamaterials composed of split ring resonators and strip wires," Physical Review E : Statistical, Nonlinear, and Soft Matter Physics, vol. 71, no. 6, Article ID 066605, 2005.

[21] A. Semichaevsky and A. Akyurtlu, "Homogenization of metamaterial-loaded substrates and superstrates for antennas," Progress in Electromagnetics Research, vol. 71, pp. 129-147, 2007.

[22] R. Mittra, "A critical look at metamaterials for antenna-related applications," Journal of Communications Technology and Electronics, vol. 52, no. 9, pp. 972-978, 2007.

[23] G. V. Eleftheriades and K. G. Balmain, Negative-Refraction Metamaterials: Fundamental Principles and Applications, John Wiley \& Sons, Princeton, NJ, USA, 2005.

[24] C. Caloz and T. Itoh, Electromagnetic Metamaterials: Transmission Line Theory and Microwave Applications, Wiley, Hoboken, NJ, USA, 2006.

[25] G. V. Eleftheriades, A. K. Iyer, and P. C. Kremer, "Planar negative refractive index media using periodically L-C loaded transmission lines," IEEE Transactions on Microwave Theory and Techniques, vol. 50, no. 12, pp. 2702-2712, 2002.

[26] G. V. Eleftheriades, O. Siddiqui, and A. K. Iyer, “Transmission line models for negative refractive index media and associated implementations without excess resonators," IEEE Microwave and Wireless Components Letters, vol. 13, no. 2, pp. 51-53, 2003.

[27] A. Sanada, C. Caloz, and T. Itoh, "Planar distributed structures with negative refractive index," IEEE Transactions on Microwave Theory and Techniques, vol. 52, no. 4, pp. 1252-1263, 2004.

[28] G. V. Eleftheriades, M. A. Antoniades, and F. Qureshi, "Antenna applications of negative-refractive-index transmission-line structures," IET Microwaves, Antennas and Propagation, vol. 1, no. 1, pp. 12-22, 2007.

[29] L. Qiang, H.-M. Lu, W. Zhao, J.-K. Wang, and B. Liu, "Simplified extended composite right/left-handed transmission line structure for dual-band applications," Progress in Electromagnetics Research Letters, vol. 15, pp. 137-144, 2010.

[30] Y. Dong and T. Itoh, "Metamaterial-based antennas," Proceedings of the IEEE, vol. 100, no. 7, pp. 2271-2285, 2012.

[31] L.-W. Li, Y.-N. Li, T. S. Yeo, J. R. Mosig, and O. J. F. Martin, "A broadband and high-gain metamaterial microstrip antenna," Applied Physics Letters, vol. 96, no. 16, pp. 164101-164103, 2010.

[32] C.-J. Lee, K. M. K. H. Leong, and T. Itoh, "Composite right/lefthanded transmission line based compact resonant antennas for RF module integration," IEEE Transactions on Antennas and Propagation, vol. 54, no. 8, pp. 2283-2291, 2006.
[33] M. A. Antoniades and G. V. Eleftheriades, "A folded-monopole model for electrically small NRI-TL metamaterial antennas," IEEE Antennas and Wireless Propagation Letters, vol. 7, pp. 425428, 2008.

[34] T. Jang, J. Choi, and S. Lim, "Compact coplanar waveguide (CPW)-fed zeroth-order resonant antennas with extended bandwidth and high efficiency on vialess single layer," IEEE Transactions on Antennas and Propagation, vol. 59, no. 2, pp. 363-372, 2011.

[35] A. Alù, F. Bilotti, N. Engheta, and L. Vegni, "Subwavelength, compact, resonant patch antennas loaded with metamaterials," IEEE Transactions on Antennas and Propagation, vol. 55, no. 1, pp. 13-25, 2007.

[36] R. W. Ziolkowski and A. Erentok, "Metamaterial-based efficient electrically small antennas," IEEE Transactions on Antennas and Propagation, vol. 54, no. 7, pp. 2113-2130, 2006.

[37] K. B. Alici and E. Ozbay, "Electrically small split ring resonator antennas," Journal of Applied Physics, vol. 101, no. 8, Article ID 083104, 2007.

[38] Y. Dong, H. Toyao, and T. Itoh, "Design and characterization of miniaturized patch antennas loaded with complementary split-ring resonators," IEEE Transactions on Antennas and Propagation, vol. 60, no. 2, pp. 772-785, 2012.

[39] Y.Zhang, J. Von Hagen, M. Younis, C. Fischer, and W. Wiesbeck, "Planar artificial magnetic conductors and patch antennas," IEEE Transactions on Antennas and Propagation, vol. 51, no. 10 I, pp. 2704-2712, 2003.

[40] H. Mosallaei and K. Sarabandi, "Antenna miniaturization and bandwidth enhancement using a reactive impedance substrate," IEEE Transactions on Antennas and Propagation, vol. 52, no. 9, pp. 2403-2414, 2004.

[41] E. Sáenz, R. Gonzalo, I. Ederra, J. C. Vardaxoglou, and P. de Maagt, "Resonant meta-surface superstrate for single and multifrequency dipole antenna arrays," IEEE Transactions on Antennas and Propagation, vol. 56, no. 4, pp. 951-960, 2008.

[42] F. Yang and Y. Rahmat-Samii, Electromagnetic Band Gap Structures in Antenna Engineering, Cambridge University Press, Cambridge, UK, 2008.

[43] N. Matsunaga, A. Sanada, and H. Kubo, "Novel twodimensional planar negative refractive index structure," IEICE Transactions on Electronics, vol. 89, no. 9, pp. 1276-1282, 2006.

[44] H. Xiong, J.-S. Hong, and Y.-H. Peng, "Impedance bandwidth and gain improvement for microstrip antenna using metamaterials," Radioengineering, vol. 21, no. 4, pp. 993-998, 2012. 

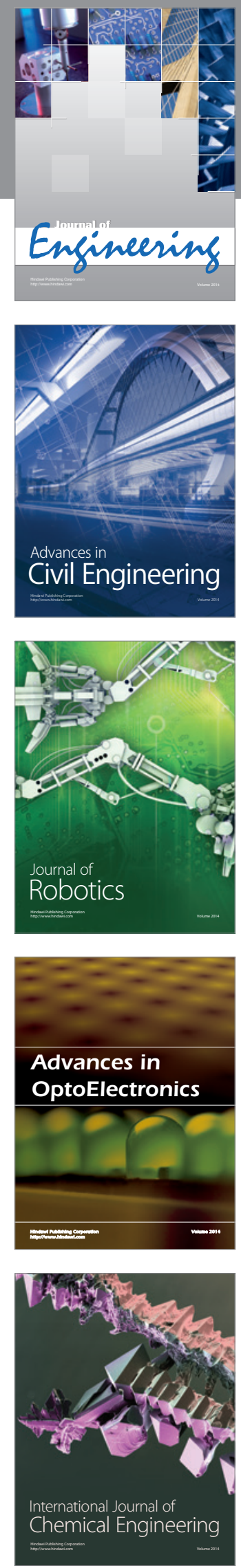

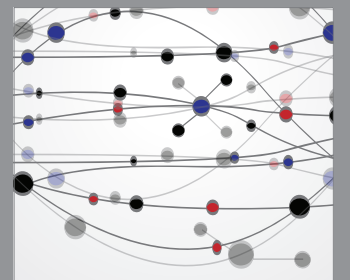

The Scientific World Journal
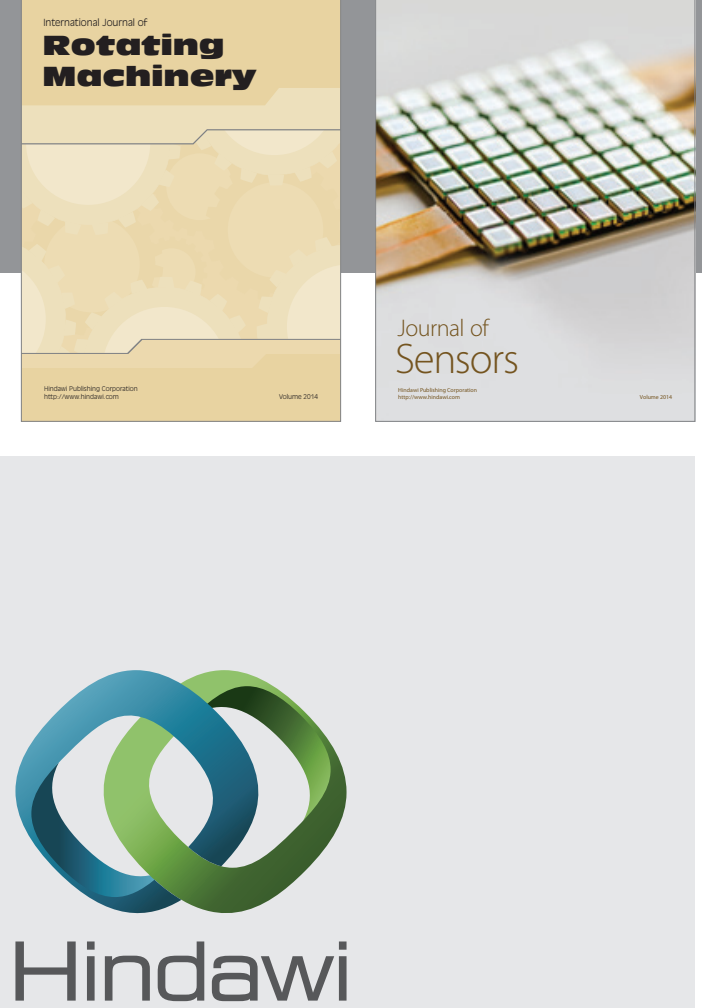

Submit your manuscripts at http://www.hindawi.com
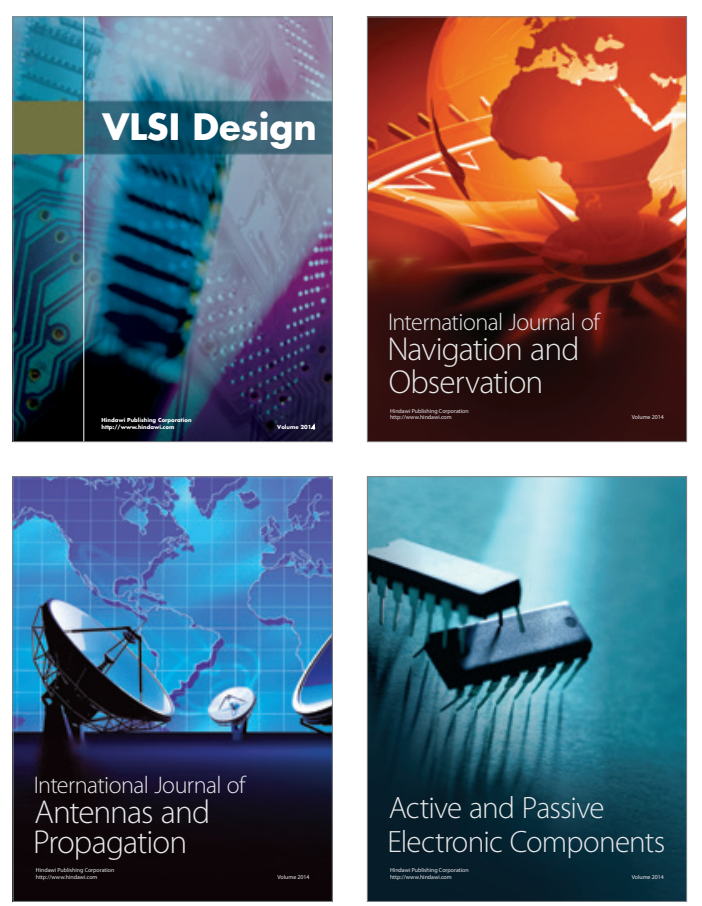
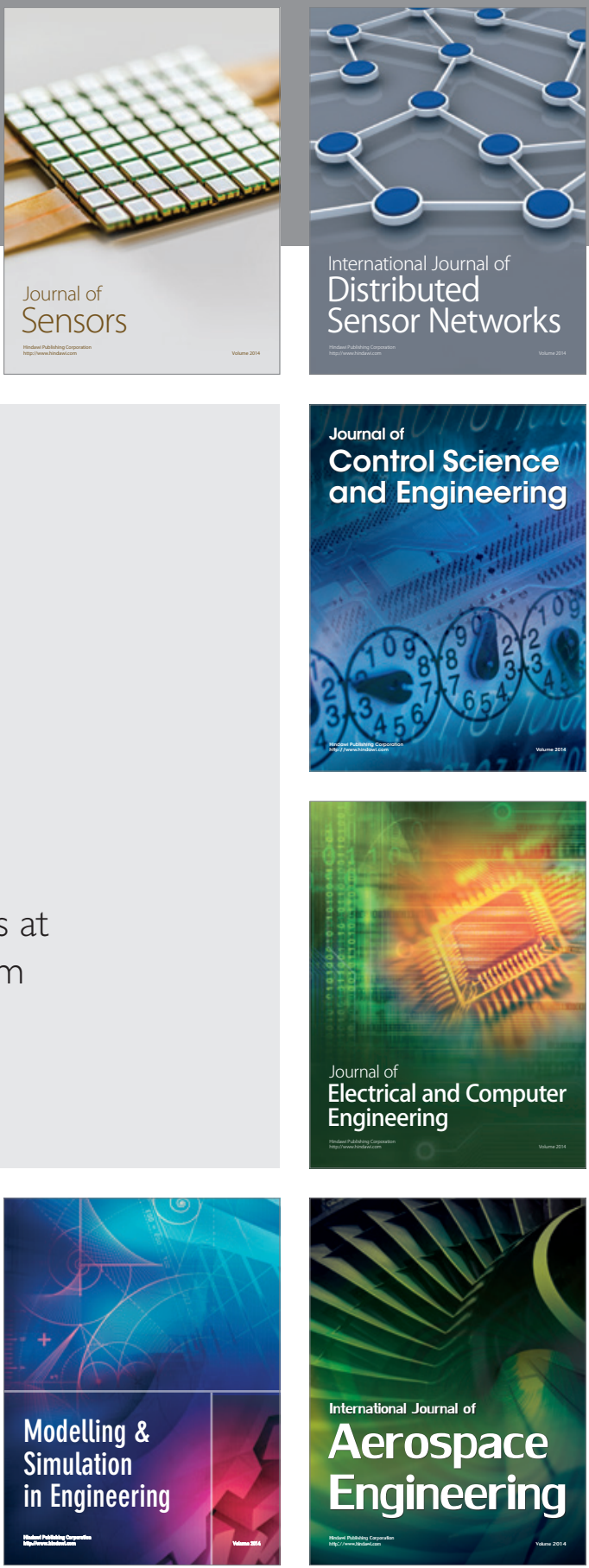

Journal of

Control Science

and Engineering
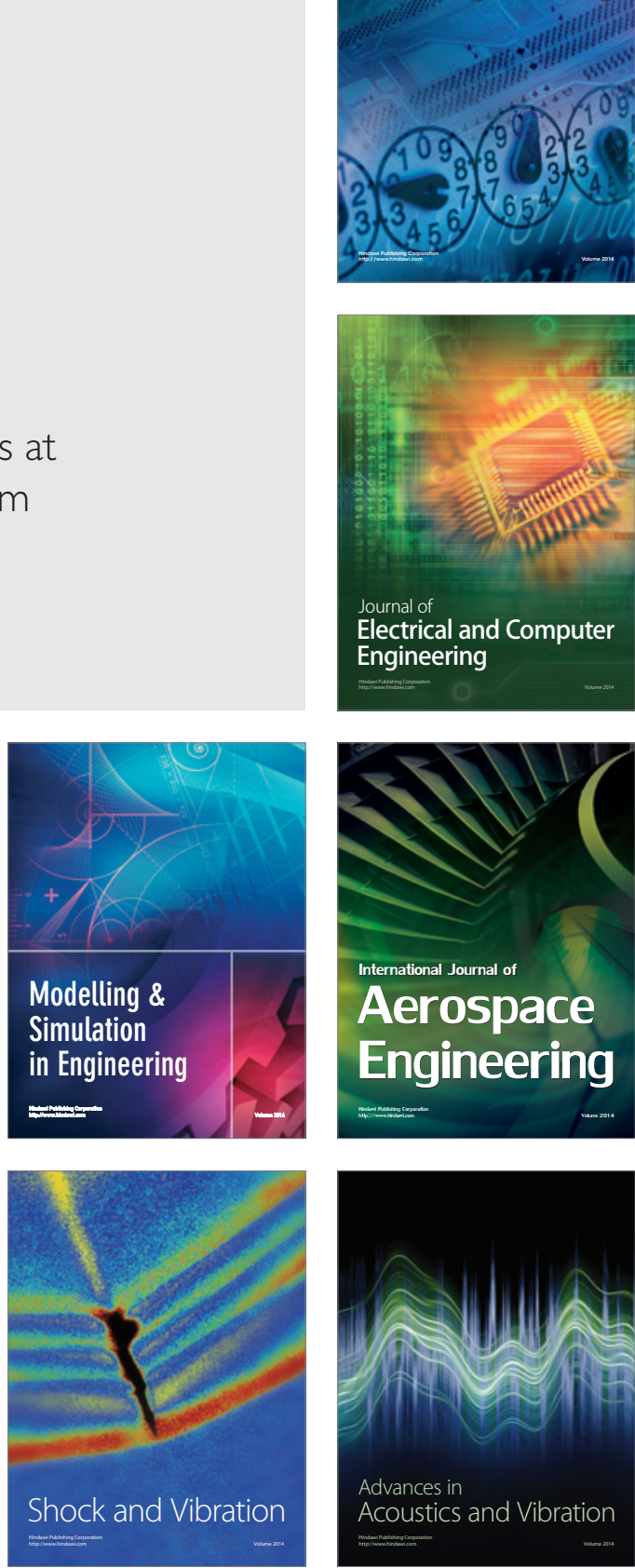